\title{
BMJ Open The NoHoW protocol: a multicentre $2 \times 2$ factorial randomised controlled trial investigating an evidence-based digital toolkit for weight loss maintenance in European adults
}

Sarah Ellen Scott (D) , ${ }^{1}$ Cristiana Duarte, ${ }^{1,2}$ Jorge Encantado, ${ }^{3}$ Elizabeth H Evans, ${ }^{4}$ Marja Harjumaa, ${ }^{5}$ Berit Lilienthal Heitmann, ${ }^{6,7}$ Graham W Horgan, ${ }^{8}$ Sofus C Larsen, ${ }^{6}$ Marta Moreira Marques, ${ }^{3,9}$ Elina Mattila, ${ }^{5}$ Marcela Matos, ${ }^{2}$ Marie-Louise Mikkelsen, ${ }^{6}$ António L Palmeira, ${ }^{3}$ Beth Pearson, ${ }^{1}$ Lauren Ramsey, ${ }^{1}$ Kirby Sainsbury, ${ }^{4}$ Inês Santos, ${ }^{3}$ Falko Sniehotta, ${ }^{4}$ Carol Stalker, ${ }^{10}$ PJ Teixeira, ${ }^{3}$ R. James Stubbs ${ }^{1}$

To cite: Scott SE, Duarte C, Encantado J, et al. The NoHoW protocol: a multicentre $2 \times 2$ factorial randomised controlled trial investigating an evidence-based digital toolkit for weight loss maintenance in European adults. BMJ Open 2019:9:e029425. doi:10.1136/ bmjopen-2019-029425

- Prepublication history and additional material for this paper are available online. To view these files, please visit the journal online (http://dx.doi. org/10.1136/bmjopen-2019029425).

Received 25 January 2019 Revised 05 August 2019 Accepted 28 August 2019

Check for updates

(C) Author(s) (or their employer(s)) 2019. Re-use permitted under CC BY. Published by BMJ.

For numbered affiliations see end of article.

Correspondence to Dr R. James Stubbs; r.j.stubbs@leeds.ac.uk

\section{ABSTRACT}

Introduction Obesity and associated diseases place a severe burden on healthcare systems. Behavioural interventions for weight loss (WL) are successful in the short term but often result in weight regain over time. Self-regulation of eating and activity behaviours may significantly enhance weight loss maintenance (WLM) and may be effectively augmented by contextual behavioural approaches to emotion regulation. The NoHoW trial tests the efficacy of a theoretically informed, evidence-based digital toolkit using a mobile-enabled website, activity trackers and Wi-Fi scales for WLM aiming to target (1) self-regulation and motivation, and (2) emotion regulation in adults who achieved clinically significant $(\geq 5 \%)$ WL in the previous 12 months (initial body mass index (BMI) $\geq 25 \mathrm{~kg} / \mathrm{m}^{2}$ ).

Methods and analysis The study is an 18-month, 3-centre, $2 \times 2$ factorial single-blind, randomised controlled trial, which recruited 1627 participants achieving $\geq 5 \%$ WL between March 2017 and March 2018. Participants are randomly allocated to one of four arms: (1) selfmonitoring only (self-weighing and activity tracker), (2) self-regulation and motivation, (3) emotion regulation or (4) combined self-regulation, motivation and emotion regulation. Participants attend four clinical investigation days at 0, 6,12 and 18 months and are instructed to use the digital toolkit for 18 weeks during the first 6 months and at their discretion for the remaining 12 months. The primary outcome is change in weight $(\mathrm{kg})$ at 12 months from baseline. Secondary outcomes are body composition (eg, bioimpedance analysis), health biomarkers (glycated haemoglobin, lipids, blood pressure, hair cortisol), dietary intake, physical activity, sleep, motivational, selfregulatory, emotion regulatory moderators/mediators of WLM, engagement, user experience, acceptability and cost-effectiveness of the interventions.

Ethics and dissemination Ethical approval was granted by Institutional Ethics Committees at the Universities of Leeds (17-0082; 27 February 2017), Lisbon (17/2016; 20 February 2017) and Capital Region of Denmark (H-
Strengths and limitations of this study

- The first project to develop and evaluate (via randomised controlled trial) an information and communications technology toolkit combining continuous tracking of physical activity and body weight with theoretically informed, evidence-based digital interventions targeting self-regulation and motivation, and emotion regulation in a $2 \times 2$ design, to target long-term weight management.

- The $2 \times 2$ factorial design is a step forward from a conventional two-armed trial to determine the effect of self-regulation/motivation, emotion regulation interventions and their interactions on weight and health outcomes.

- Analyses of primary outcomes will test the hypotheses that self-regulation of energy balance behaviours improves longer term weight outcomes and that emotion regulation strategies help prevent weight relapse. Moderator analysis will determine if participant psychological/behavioural profiles predict response to self-regulation or emotion regulation interventions. Mediation analyses will examine mechanisms of action by which self-regulation and emotion regulation-based approaches affect energy balance behaviours, weight and health.

- Participants are blinded to study allocation, but they can access similar interventions from other real-world sources.

- There was no zero-intervetion control. The active control arm includes self-monitoring of physical activity and weight but the inclusion of the Fitbit devices across all arms potentially contaminates or diminishes the effect of self-regulatory components. Social support was excluded as a potential contaminant of the digital intervention.

16030495, 8 March 2017). Results will be published in scientific journals.

Trial registration number ISRCTN88405328. 


\section{INTRODUCTION}

Overweight, obesity and associated diseases are key societal challenges. Obesity prevalence accounts for 3\%-8\% of health costs and 10\%-13\% of deaths in Europe. ${ }^{1}$ This translates into $€ 59$ billion a year in direct, and an estimated $€ 118-€ 236$ billion in indirect, healthcare costs. ${ }^{2}$ These costs are set to rise in parallel with obesity prevalence. ${ }^{3}$ Approximately $40 \%$ of the adult population in Europe report engaging in at least one weight control attempt in the previous 12 months. ${ }^{4}$ Existing community-based programme support initial weight loss (WL) but are subject to high attrition or weight regain, limiting longer term effectiveness. ${ }^{56}$ The obesogenic environment and asymmetry of human energy balance (EB) regulation facilitates weight gain, while society criticises people suffering from overweight and obesity, leading to stress and negative emotions, which can undermine weight loss maintenance (WLM) attempts. ${ }^{78}$ Effective support for WLM or the prevention of weight regain needs to address sustained changes in EB behaviours (dietary, physical activity and weight control) and additional emotional/ psychosocial challenges that may undermine planned healthy behaviours.

\section{Self-regulation and motivation}

Core features of effective WLM interventions include behaviour change techniques in line with self-regulation theories such as (1) self-monitoring of weight and behaviour; (2) goal-setting: agreement of clear weight targets/trigger points for weight control efforts; (3) feedback on behaviour and weight; (4) action plans for weight control through dietary and physical activity behaviours; and (5) plans to cope with risk factors for weight regain and relapse prevention (eg, problem solving) ${ }^{9-15}$ Further, self-determined autonomous motivation has been consistently associated with change in EB behaviours for obesity management. ${ }^{16}{ }^{17}$ Interventions rooted in Self-Determination Theory may offer a promising approach to sustained EB and weight control. ${ }^{12}{ }^{17}$ A recent systematic review exploring psychological mediators of sustained effects in diet and lifestyle obesity interventions found that higher levels of autonomous motivation, self-efficacy, self-regulation skills, flexible eating restraint and positive body image were mediators of medium-term to long-term weight control. ${ }^{18}$ The combination of motivational and volitional processes of $\mathrm{EB}$ behaviours can contribute to effective WLM; that is, it appears those who are adequately motivated and who implement intentions tend to both initiate and maintain such changes. ${ }^{19-21}$ However, physical activity and dietary interventions based on current behaviour change theories characteristically achieve significant, but small and heterogeneous effects. ${ }^{22}$ Additional psychological processes, such as emotion regulation, can potentially have incremental beneficial effects on the behavioural changes that promote WLM. ${ }^{23}$

\section{Emotion regulation}

Individuals with obesity or those trying to lose weight can experience increased stress, depression, anxiety and weight-related stigma ${ }^{24} 25$ which may undermine weight control attempts. Repeated attempts at WL followed by weight regain can have a negative emotional impact, leading to self-critical thoughts and negative emotions. For some, 'comfort eating' may be a means of coping with these negative experiences, potentially derailing strategies of planned behaviour. ${ }^{26-28}$ Relapse prevention models include contextual behavioural (CB)-based emotion regulation strategies such as compassion, acceptance and mindfulness. ${ }^{29-31}$ Evidence suggests that mindfulness and acceptance of undesired internal experiences (eg, negative emotions or self-criticism) and compassion for the self and others may help some individuals adopt adaptive behaviours that are congruent with their weight goals or values. ${ }^{32-36}$ Mindfulness-based interventions show promise in changing obesity-related eating behaviours (eg, binge eating and emotional eating), lowering depression levels and improving WLM. ${ }^{37}$ Augmenting current self-regulatory approaches with $\mathrm{CB}$ emotion regulatory approaches could lead to more effective WLM. ${ }^{32-36}$

\section{Digital behaviour change interventions}

Recently, there has been interest in digital interventions to promote and maintain health. ${ }^{38}$ Digital solutions, such as smartphone applications and wearables, can be effective in supporting WLM if they are evidence-based, apply a choice of behaviour change techniques with individualised feedback, ${ }^{22}{ }^{39}{ }^{40}$ encourage the use of self-regulatory techniques (eg, self-monitoring) and develop intrinsic motivation. ${ }^{41}$ Digital solutions are potentially cost-effective and scalable to large populations, which could engage citizens in healthcare innovations that are convenient and effective for weight management in the face of limited public budgets. ${ }^{42}$ To date, most commonly available weight management applications miss key evidencebased approaches for longer term behaviour change. ${ }^{43} 44$

\section{Study objectives and hypotheses}

The effectiveness of long-term evidence-based digital interventions and strategies to support WLM is limited. ${ }^{45}$ The study's primary objectives are to evaluate whether using a new digital toolkit is effective for WLM by improving (1) self-regulation and motivation, (2) emotion regulation or (3) these factors in combination, compared with (4) control (generic toolkit content, regular self-weighing and Fitbit use), in 1627 participants at three European centres. We hypothesise that the combined intervention will be more effective for WLM compared with the self-regulatory or emotion regulatory interventions alone, which will also be more effective than the control group.

Secondary objectives are to determine (1) how the intervention affects health markers (eg, levels of glycated haemoglobin (HbA1c)) and body composition; (2) examine the intervention impacts on physical activity, sleep, dietary intake, depression, anxiety, stress, quality of life and well-being; (3) examine mediators of WLM, such as self-regulation (eg, planning capacity), motivation (eg, autonomous motivation) and emotion regulation 

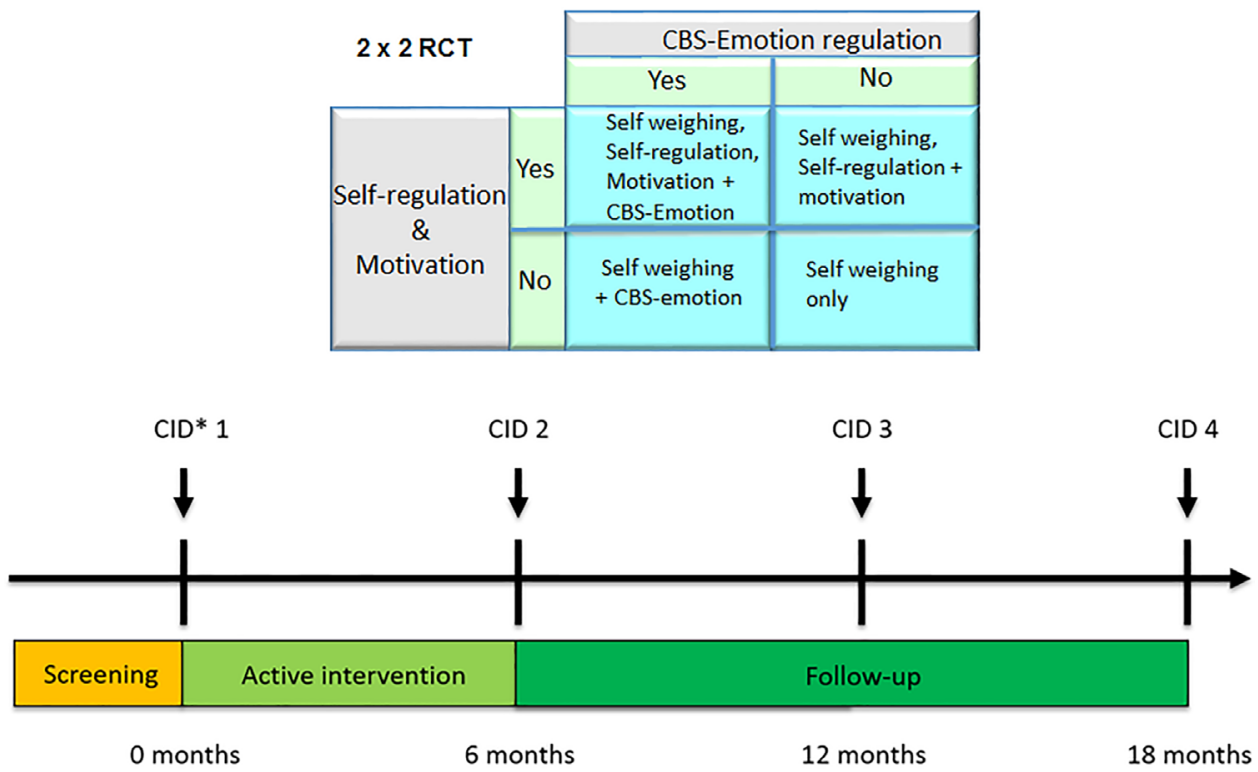

Baseline

Primary outcomes ${ }^{\dagger}$

${ }^{*}$ CID, Clinical Investigation day.

${ }^{+}$All measures are taken at CID 1-4 with the exception of the biomarkers which are taken at 0 and 12 months only. Process evaluation measures are taken at 1, 3, 6 and 12 months and focus groups at 6 months.

Figure 1 Trial design. RCT, randomised controlled trial.

processes (eg, self-compassion); (4) analyse baseline moderators (eg, gender, body mass index (BMI)) to identify participants who are more responsive to motivational and behavioural self-regulatory or CB-emotion regulatory approaches for WLM; (5) conduct quantitative and qualitative assessment of user-experience, acceptability, engagement and dropout; (6) examine intervention cost-effectiveness; and (7) to determine the efficacy of individualised feedback from data on physical activity self-tracking.

\section{METHODS}

\section{Trial design and setting}

The NoHoW trial is a three-centre (University of Leeds (UK), The Parker Institute (Denmark) and University of Lisbon (Portugal)) 2×2 factorial, randomised, singleblind, controlled trial testing the proof-of-concept of a digital toolkit for WLM. The study duration is 18 months with a follow-up at 6,12 and 18 months postbaseline (figure 1). The study is conducted between March 2017 and September 2019 in academic research institutes. In total, 1627 participants ( 542 per centre) were enrolled and randomised into one of four intervention arms: (1) active control arm (consisting of generic toolkit content, self-weighing and activity trackers, only); (2) self-regulation and motivation arm; (3) emotion regulation arm; and (4) combined motivation/self-regulation and emotion regulation arm (figure 1). The protocol is harmonised across trial centres using Good Clinical Practice guidance, research-grade translation/backtranslation of trial materials, two training workshops and weekly trial management meetings. The trial registration data set is displayed in table 1. The final protocol (V2.1 20/09/2017) was approved by the Trial Steering Committee (TSC) and adheres to the Standard Protocol Items: Recommendations for Interventional Trials guidelines. ${ }^{46}$

\section{Participants}

Table 1 describes the inclusion/exclusion criteria for the trial. Prior to randomisation, potential participants are asked to provide documented verification (by a health professional, WL counsellor/friend, WL programme record booklet, diary or smartphone app or before/after photographs ${ }^{47}$ ) that they have achieved a clinically significant WL of $>5 \%$ during the previous 12 months.

\section{Study recruitment}

The study recruitment and intervention schedule is outlined in table 2. Centre-specific recruitment strategies were adopted for 12 months (March 2017-March 2018) and included commercial WL programme (UK, Slimming World); the Copenhagen Municipality weight management services, Dieticians from the Danish Association for Dieticians and commercial slimming companies (eg, Sense, Henrik Duer and Per Nielsen); registered clinical dieticians/nutritionists who provide weight management services in Lisbon; leisure centres; and local/national media coverage and advertisements. All participants were directed to country-specific recruitment websites (http://uk.nohow.eu; http://dk.nohow. eu; http://nohow.fmh.ulisboa.pt) and completed an 
Table 1 WHO trial registration data set

\begin{tabular}{|c|c|}
\hline Data category & Information \\
\hline $\begin{array}{l}\text { Primary registry and trial identifying } \\
\text { number }\end{array}$ & ISRCTN: 88405328 \\
\hline Date of registration in primary registry & $16 / 12 / 2016$ \\
\hline Secondary identifying numbers & H2020-EU.3.1 Grant agreement ID: 643309 \\
\hline $\begin{array}{l}\text { Source(s) of monetary or material } \\
\text { support }\end{array}$ & European Commission, Horizon 2020-EU Framework Programme for Research and Innovation \\
\hline Primary sponsor & University of Leeds, Leeds, UK \\
\hline Secondary sponsor(s) & $\begin{array}{l}\text { The Parker Institute, Copenhagen, Denmark } \\
\text { University of Lisbon, Lisbon, Portugal }\end{array}$ \\
\hline Contact for public queries & $\begin{array}{l}\text { Prof. James Stubbs (r.j.stubbs@leeds.ac.uk) } \\
\text { School of Psychology } \\
\text { Faculty of Medicine and Health } \\
\text { University of Leeds } \\
\text { Leeds } \\
\text { LS2 9JT } \\
\text { UK } \\
+44(0) 1133433476\end{array}$ \\
\hline Contact for scientific queries & Professor James Stubbs (r.j.stubbs@leeds.ac.uk) \\
\hline Public title & NoHoW: Evidence-based ICT tools for weight loss maintenance \\
\hline Scientific title & $\begin{array}{l}\text { A } 2 \times 2 \text { randomised controlled trial to evaluate the effectiveness of evidence-based ICT behaviour } \\
\text { change tools for weight loss maintenance in overweight/obese adults after clinically significant } \\
\text { weight loss. }\end{array}$ \\
\hline Countries of recruitment & $\begin{array}{l}\text { 1. University of Leeds (UK). } \\
\text { 2. The Parker Institute (Denmark). } \\
\text { 3. University of Lisbon (Portugal). }\end{array}$ \\
\hline
\end{tabular}

Health condition(s) or problem(s) studied Adults aged 18 or older who have intentionally lost at least $5 \%$ of their body weight in the last 12 months, and initially had a BMI of 25 or more.

Intervention(s)

Participants are assigned to one of four interventions:

1. Self-monitoring only (self-weighing and activity tracker).

2. Self-regulation and motivation.

3. Contextual behavioural science-based emotion regulation (CBS-emotion regulation).

4. Combined self-regulation, motivation and CBS-emotion regulation.

Key inclusion and exclusion criteria Inclusion criteria:

1. Aged 18 years or older.

2. BMl (prior to weight loss) of $\geq 25 \mathrm{~kg} / \mathrm{m}^{2}$.

3. Verification of $\geq 5 \%$ of weight loss in the last 12 months and remain $5 \%$ below their highest weight.

4. Ability to use a smartphone and have access to smart phone, tablet or computer with internet access and Wi-Fi.

5. Ability to use standing scales for weight measurements and must not be over $150 \mathrm{~kg}$ (the scale limit $158 \mathrm{~kg}$ )

Exclusion criteria:

1. Inability to give informed consent.

2. Lost weight due to illness or surgical procedures, including bariatric procedures.

3. Pregnant or breastfeeding.

4. Involvement in another research intervention study that confound with the aims of NoHoW (excluding local health interventions and weight management services).

5. Inability to follow written material or telephone conversations in the English, Danish or Portuguese language (depending on the centre).

6. Diagnosed with an eating disorder (eg, anorexia nervosa, bulimia nervosa or purging disorder).

7. Diagnosed with any condition that may interfere with increasing mild to moderate physical activities and which is unstable (ie, untreated or unable to be controlled by medication).

8. Recent diagnosis with type 1 diabetes.

9. Extensive planned travel, that is, moving away or long-term travel abroad (eg, more than 4 weeks).

10. Living in the same household as existing participant in the trial.

\begin{tabular}{ll} 
Study type & Interventional, $2 \times 2$ factorial randomised controlled trial. \\
Date of first enrolment & 20 March 2017 \\
\hline Target sample size & 1600 \\
Recruitment status & Completed (31/03/2018), no longer recruiting. \\
\hline
\end{tabular}


Table 1 Continued

\begin{tabular}{ll}
\hline Data category & Information \\
\hline Primary outcome(s) & Weight (kg) \\
Key secondary outcomes & 1. Body composition: (eg, fat mass and fat-free mass) and waist/hip ratio. \\
& 2. Biomarkers: blood pressure, heart rate, cortisol (hair samples), HbA1c, cholesterol. \\
3. Behavioural: physical activity, sleep and dietary intake. & 4. Psychological moderators and mediators of change for: self-regulation and motivation (eg, action \\
& planning and coping, self-efficacy); CBS-emotion regulation (eg, weight focused self-criticism, \\
& five dimensions of mindfulness); stress management (eg, perceived stress scale, depression, \\
& anxiety and stress); and quality of life and well-being. \\
5. Process evaluation & 6. Cost-effectiveness analysis
\end{tabular}

BMI, body mass index; ICT, information and communications technology.

online eligibility screener using Qualtrics. The eligibility screener categorised respondents as eligible, potentially eligible (wait-list) and ineligible. Eligible individuals were contacted for a telephone screening interview, provided with study information, medical history questions and the Physical Activity Readiness Questionnaire. ${ }^{48}$ Eligible participants were invited to a clinical investigation day (CID) where informed consent was obtained by research staff before randomisation (online supplementary file 1). Participants are reconsented before each subsequent visit and excluded if they become ineligible.

\section{Public involvement}

Members of the public that matched the study's inclusion criteria at the three trial centres were consulted via a survey to inform the recruitment strategies, trial conduct, questionnaires, assessment of burden and reviewing or testing the intervention content at all trial centres. During the toolkit development stage, the public were involved in two ways. First, a panel of 10 people, matching the trial criteria, tested the toolkit V.1 written in English for 1 month in the UK. A mixed methods approach was used for the analysis of the data assessing technical maturity of the intervention and user experiences, through questionnaires (quantitative data), interviews (qualitative data), TK usage $\log$ data and data from wireless scales and activity trackers (Fitbit). These analyses will be published in a separate paper. The results were used to inform toolkit V.2. Toolkit V.2 was then tested for 18 months using a panel of 30 people (10 in each centre) starting 3 months before the launch of the trial. Trial staff regularly collaborated with the panel to identify technological glitches that could be corrected early on so as not to impact on the main trial participants.

\section{Randomisation and blinding}

Participants were randomly allocated to treatment arms by researchers using an online trial administration portal. Adaptive stratified sampling using minimisation ${ }^{49}$ was embedded in the portal using the R-programme ( $\mathrm{R}$ Foundation for Statistical Computing, Vienna, Austria) by the study statistician GH. This minimises differences

Table 2 Study recruitment and intervention schedule (Standard Protocol Items: Recommendations for Interventional Trials 2013 guidelines)

\begin{tabular}{|c|c|c|c|c|c|}
\hline \multirow[b]{3}{*}{ TIME POINT } & \multicolumn{5}{|c|}{ Study duration } \\
\hline & Recruitment & Baseline & 6 months & 12 months & 18 months \\
\hline & to & t1 & t2 & t3 & t4 \\
\hline \multicolumn{6}{|l|}{ RECRUITMENT } \\
\hline Screening & $\mathrm{x}$ & & & & \\
\hline Informed consent & & $x$ & & & \\
\hline Randomisation and allocation & & $\mathrm{x}$ & & & \\
\hline \multicolumn{6}{|l|}{ INTERVENTION } \\
\hline Fitbit device training & & $\mathrm{x}$ & & & \\
\hline Intake24 (food diary) training & & $x$ & & & \\
\hline $\begin{array}{l}\text { Toolkit training (after } \\
\text { randomisation) }\end{array}$ & & $x$ & & & \\
\hline Arms $2-4$ active intervention & & $\bullet----$ & & & \\
\hline Arms 2-4 follow-up & & & $\bullet----$ & ------ & --- \\
\hline Arm 1 (control) intervention & & ---- & ----- & ------ & --- \\
\hline
\end{tabular}


in age, $\mathrm{WL}$ in the previous 12 months and current BMI between treatment arms and stratified by gender and centre. Participants were blinded to study allocation. The research team were not blinded due to the need to train participants in arm-specific toolkit versions. The statistician $(\mathrm{GH})$ is independent from data collection but not blinded during analyses due to inclusion of the arm allocation number. Independent researchers analysing hair cortisol are blinded to group allocation. No preliminary or ancillary analysis of outcome data between intervention arms were conducted prior to data-lock (12 months).

\section{Interventions}

The toolkit is a mobile-enabled website, delivered as a portfolio of embedded tracking technologies, mini-apps and web resources (figure 2) accessible via computer, tablet

\section{NHoW}
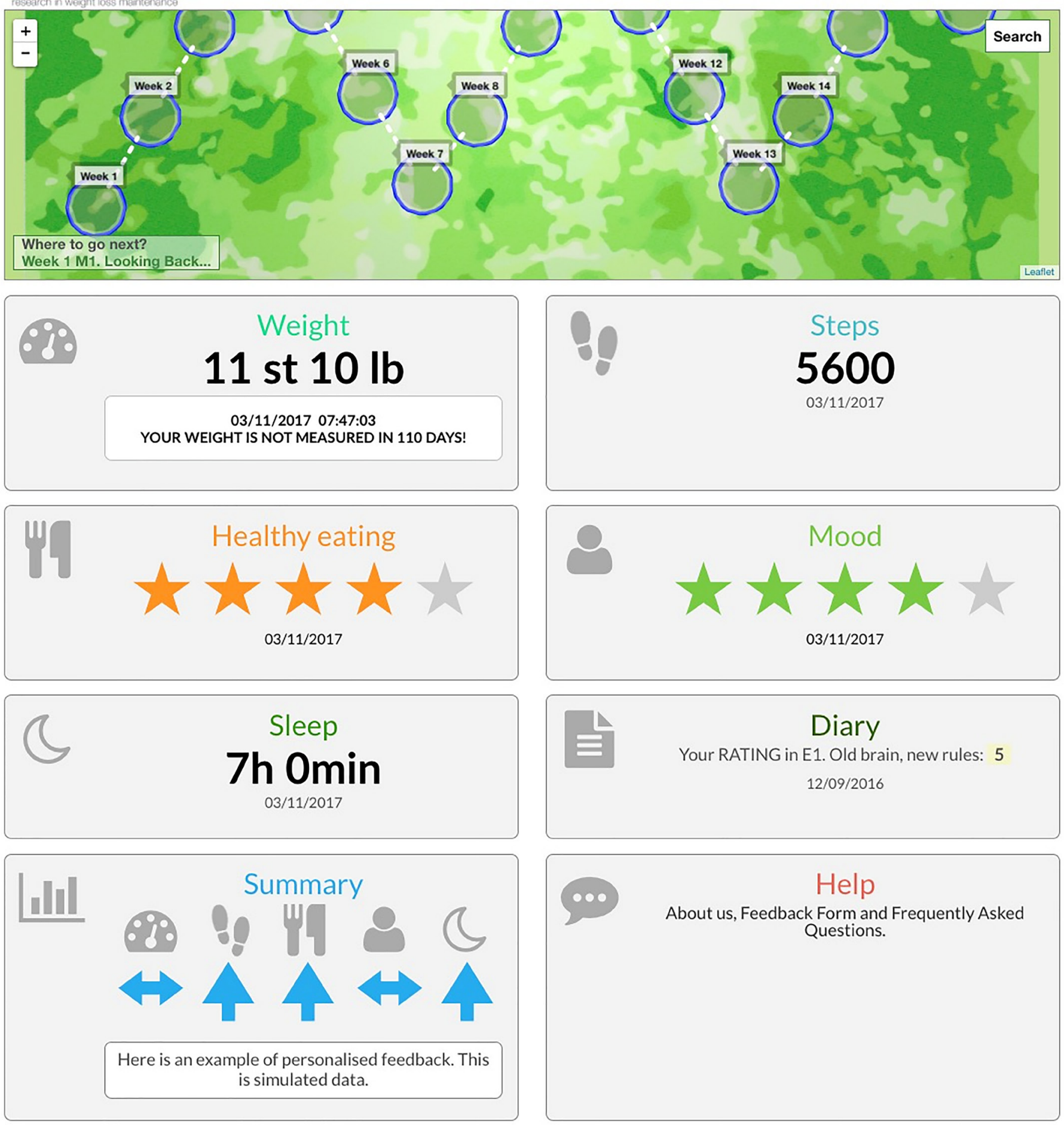

Figure 2 Toolkit example (arm 4). 
and mobile phone. The tracking technologies consist of an activity and sleep tracker (Fitbit Charge 2) and smart wireless body weight scale (Fitbit Aria). Participants were asked to wear the Fitbit Charge 2 at all times and to weigh themselves $\geq$ twice weekly. All arms include instructions for self-weighing and use of activity trackers. Arms 2 and 4 include individualised weekly feedback about weight trajectories. Guidance for complex interventions was followed during the toolkit development specifying the intervention logic models and theory-driven behaviour change techniques, ${ }^{50}$ which will be detailed in a separate publication.

\section{Arm 1 (active control, self-monitoring, only)}

The active control arm includes a toolkit dashboard containing weight, steps and sleep graphs using data from the Fitbit devices, healthy eating and mood data (self-scored on a five-point scale), a diary to populate with notes from these five tiles and a help tile. The content is generic and does not relate to any theoretically informed, evidence-based behaviour change approaches.

\section{Arms 2-4}

After randomisation, participants received standardised training from researchers during the first CID, specific to their arm allocation. Trial arms 2-4 include the same components as arm 1 but participants also receive a personal virtual road map (the 'NoHoW Journey') with intermediate destinations represented in that map by circles, each comprised of evidence-based theoretically informed thematic modules targeting self-regulation and motivation (arm 2), emotion regulation (arm 3) and combined self-regulation and emotion regulation (arm 4). Arm 4 includes the same number of thematic modules as arms 2 and 3. Each weekly module opens into submodules. The active intervention involves 18 weekly sessions delivered throughout a 6 -month period. Users are encouraged to complete $\geq 1$ submodule per week following the sequence in the road map, consisting of videos/audios, interactive graphs, text, images, questionnaires and mini-apps. Weekly arm-specific emails are sent during the active 6-month intervention phase, which provide WLM information, suggestions and reminders to visit specific content. The toolkit automatically sends an email notifying participants to slow down if they complete three sessions ahead of schedule. After 6 months, they are encouraged to revisit the toolkit for a further 12 months at their discretion.

Individualised feedback is a component of the self-regulation and motivation arms (arms 2 and 4) automatically provided by the toolkit in response to the individual's weight trajectory. Feedback is generated from the data extracted from three sources: (1) weight from the Fitbit Aria scales; (2) activity and sleep from the Fitbit Charge 2; and (3) user engagement with the toolkit, including day of the week. After daily usage patterns are analysed, feedback is displayed in the toolkit consisting of one of a set of short statements (eg, "your weight management appears better when you are more active").

\section{Adherence}

The toolkit sends automated weekly emails to participants to encourage engagement, prompt log-in and recommends sessions to complete (arms 2-4 only). The control group receives the same number of emails but with links to generic content (eg, topical diet/health information). Intervention adherence is continuously monitored by collecting information on the number of days since users last logged into the toolkit and the number of days since the last synchronisation between the Fitbit devices and data-hub. If no activity is detected after 21, 28 and 35 days, three attempts are made to contact participants by email. After 42 days of no activity, researchers telephone participants to resolve issues. Reasons for no activity are recorded.

\section{Assessments}

The primary outcome is change in weight $(\mathrm{kg})$ between baseline and 12 months (primary endpoint). Follow-up measures for WLM are completed at month 18 for comparability with other longitudinal studies. ${ }^{22}$ All physical measurements are taken after a 10-12 hour overnight fast by trained research staff. Measures are taken at all four time points (baseline, 6, 12 and 18 months) except HbAlc, lipids and hair cortisol (baseline and 12 months only) and process evaluation measures (1, 3, 6 and 12 months only). The study schedule and information for all measures is in online supplementary file 2 .

\section{Participant retention}

Randomised participants are contacted at least 2 weeks before each visit and sent an email/text reminder. If participants deviate from planned assessments, three attempts are made to contact them by phone, email and a final written letter across 1 month. At least weight (primary outcome) is obtained if possible.

\section{Primary outcome}

Change in weight $(\mathrm{kg})$ at 12 months from baseline

Body weight $( \pm 0.1 \mathrm{~kg})$ is measured using a Seca $704 \mathrm{~s}$ instrument (SECA, Germany) in participants wearing light clothing. Participants are asked to weigh themselves at least twice weekly, in the morning after voiding and before eating using the Fitbit Aria scales for 18 months.

\section{Secondary outcome measures}

Body composition and height

Multifrequency whole bioimpedance spectroscopy is measured by ImpediMed (SFB7, Queensland, Australia), which measures impedance over a spectrum of frequencies for the estimation of body composition. Hanai mixture theory equations and standard resistivity constants $^{51}$ are used to calculate fluid volumes to estimate total body water. Height $( \pm 0.1 \mathrm{~cm})$ is measured with participants barefoot, using a Seca $704 \mathrm{~s}$ instrument (SECA, Germany). See online supplementary file 2. 


\section{Waist-hip ratio}

Waist and hip measurements are taken according to the WHO's guidance. A tape measure is used to record the hip and waist circumference to the nearest centimetre. ${ }^{52}$ The waist-hip ratio is calculated by dividing hip and waist circumference.

\section{Biomarkers}

\section{$\mathrm{HbA1c}$ and cholesterol}

Fasted capillary blood samples are collected to determine $\mathrm{HbAlc}(\mathrm{mmol} / \mathrm{mol}, \%)$, estimated average glucose (eAG, $\mathrm{mol} / \mathrm{L}$ ) and full lipid profiles, including total cholesterol, low-density lipoprotein, high-density lipoprotein (HDL), triglycerides, non-HDL and cholesterol/HDL (mmol/L) assayed using a benchtop analyser (Alere Afinion AS100 Analyser, Alere, Stockport, UK). ${ }^{53}$

\section{Blood pressure heart rate}

Systolic and diastolic blood pressure (BP) and resting heart rate (HR) (Microlife BP A2 Basic, Gentle Technology, Microlife, Clearwater, FL, USA,) are measured three times with the participant at rest in the sitting position. The mean value is recorded.

\section{Hair cortisol}

Hair samples are collected by cutting a small tuft of hair close to the scalp to measure the stress hormone, cortisol, and analysed following a previously described protocol ${ }^{54}$ (online supplementary file 2).

\section{Physical activity}

Physical activity is measured by the self-reported International Physical Activity Questionnaire (IPAQ) ${ }^{55}$ and Activity Choice Index (ACI) ${ }^{56}$ Minute-by-minute physical activity data and heart rate are measured by the Fitbit Charge 2 for the study duration.

\section{Sleep quality and quantity}

The Fitbit Charge 2 estimates sleep quantity (hours/ minutes) and quality (stages of sleep). Data are collected throughout the trial.

\section{Dietary intake and eating behaviour}

Four consecutive 24-hour-web-based dietary recalls, including at least 1 weekend day, are collected within 7 days of each visit using INTAKE $24 .^{57}$ Psychometric measures of eating behaviour are assessed at each visit using the Three Factor Eating Questionnaire-51, ${ }^{58}$ the Problematic Eating Patterns Scale (unpublished scale), Eating in the Absence of Hunger Scale, ${ }^{59}$ Intuitive Eating Scale- $2^{60}$ and the Binge Eating Scale. ${ }^{61}$

\section{Well-being and quality of life}

Quality of life and well-being are assessed using the 5-level EQ5D (EQ5D-5L) ${ }^{62}$ and the Warwick-Edinburgh WellBeing Scale, ${ }^{63}$ respectively.

\section{Self-regulation}

Self-regulation is measured using the Action Planning and Coping Planning Scales ${ }^{64}$ and Action Control Scale. ${ }^{65}$
Motivation

Satisfaction of psychological needs are assessed using the Basic Psychological Needs and Frustrations Scale. ${ }^{66}$ Quality of goal contents is assessed using the Goal Content for Weight Loss Maintenance Scale. ${ }^{6768}$ Autonomous and controlled motivation are assessed using the Behavioural Regulation in Exercise Questionnaire (BREQ-3), ${ }^{69}$ Regulation of Eating Behaviour Scale (REBS) ${ }^{70}$ and Regulations for Weight Management Scale (adapted from the REBS). ${ }^{70}$

\section{Self-efficacy}

Self-efficacy is assessed using the Self-Efficacy for Weight Maintenance Scale. $^{71}$

\section{Emotion regulation and stress management}

CB-emotion regulation and stress management constructs are measured using the Weight Focused Self-Criticism/ Self-Reassurance Scale, ${ }^{72}$ Weight Focused External Shame Scale, ${ }^{73}$ the Compassion Engagement and Actions Scales, ${ }^{75}$ Body Image Acceptance and Action Questionnaire, ${ }^{76}$ Engaged Living Scale, ${ }^{77}$ Mindful Attention Awareness Scale, ${ }^{78}$ adapted Difficulties in Emotion Regulation Scale $^{79}$ and Experiences Scales (decentering subscale only). ${ }^{80}$ Perceived stress is measured by the Perceived Stress Scale. ${ }^{81}$ Anxiety and depression are measured using the Depression \& Anxiety Stress Scales. ${ }^{82}$

\section{Demographics, weight history and participant traits}

A profiling questionnaire, adapted from the DiOGenes study, ${ }^{83}$ is administered at baseline only to measure trait-specific moderators of WLM including the following demographics: age, gender, country, education, marital status, employment and income. Also, smoking and alcohol use and number of times giving birth are recorded. Weight history includes height, current and highest weight in the last 12 months, successful and unsuccessful WL attempts and strategies, and psychological impact of living in a food abundant environment (the Power of Food Scale). ${ }^{84}$ Self-determination traits are assessed using the Index of Autonomy Functioning ${ }^{85}$ and Aspirations Index, ${ }^{86}$ and self-regulation of appetite, hunger and satiety cues by the Intuitive Eating Scale-1. ${ }^{87}$ Relapse prevention capability is measured using an adapted Relapse Prevention Scale. ${ }^{88}$

\section{Process evaluation and user experience}

Questionnaires adapted from the Technology Acceptance Model for Mobile Services (TAMM) ${ }^{89}$ are administered by email to assess reasons for engagement/dropout and user-experience (eg, perceived ease-of-use and adoption, perceived value, trust, acceptability and usability). Digital literacy is assessed at 1 month using the eHealth Literacy Scale (eHEALS).$^{90}$ Twelve focus groups were conducted (four in each country) at 6 months, by independent researchers, to thematically explore contextual factors (eg, influence of wider social networks), user experience and the toolkit use context in the three countries. ${ }^{91}$ Metadata on reach (participant characteristics), dose, fidelity and engagement with the toolkit are extracted from log 
files (online supplementary file 2). Methods and analyses will be detailed in separate publications.

\section{Data management}

A NoHoW data-hub was developed by the James Hutton Institute (Edinburgh) and led by GH, which collates, monitors and stores anonymised data securely from each centre. Data from each centre are entered into trial management software (Easy Trial: www.easytrial. net). Quality checks are performed by all centres and reviewed during weekly trial management meetings. Separate publications will detail the technologies to host the toolkit, and the ICT architecture linking the toolkit, trial administration portal, trial management software and NoHoW data-hub.

\section{Data analysis}

The $2 \times 2$ factorial design will be used in a model to investigate links between outcomes, and the extent of mediation or moderation by covariates. For continuous outcomes, linear regression models will be used. The effects of different treatment arms will be presented with 95\% CI of coefficients and $p$ values. For analyses that do not meet model assumptions, outcomes will be transformed prior to inferential comparisons. For categorical outcomes, logistic regression or categorical response models such as ordinal or multinomial regression will be used. Treatment effects will be presented as ORs, 95\% CIs and $\mathrm{p}$ values. Missing values will be imputed using multiple imputation as part of an intention-to-treat (ITT) analysis.

To determine the mechanisms that underlie intervention efficacy, linear models are used to test whether the treatment (independent variable) causes a change in the potential mechanisms of change (mediators), which, in turn, cause a change in the primary and secondary outcomes (dependent variables). Models also investigate the effects of covariates that may moderate the effect of the interventions. Although a repeated measures/ mixed model might potentially detect treatment effects not apparent when 6-month and 12-month outcomes are considered separately, our choice was driven by the primary role of the 12-month outcome. To be considered worthwhile, a WL or maintenance effect of treatment must be detectable at 12 months, whatever might happen during the time interval between then and baseline. Because of this, we chose to define the primary outcome and its analysis at 12 months alone.

For the primary outcome of weight, we intend to present results from an analysis of completers, of compliant participants and of the ITT population. Baseline and last observation carried forward are likely to be optimistic for dropouts, implying full WLM. So, for these dropouts, we will examine analyses which assume regain of all or a range of proportions of the weight lost since highest weight (in the 12 months before enrolment, for which all participants have provided evidence). Again, assuming return to baseline may be optimistic, but we do not have values at the time of greatest weight. Accordingly, we plan multiple imputation based on the imputed weight changes described above, using also centre and gender. This is not available for other health outcomes, such as HbAlc or blood pressure.

Mediation is assessed where change in weight, as represented by a categorical endpoint (maintenance vs weight regain), is the dependent variable, intervention group is the predictor variable, and self-regulatory, emotion regulatory and other psychosocial variables are mediators. Hybrid models, combining latent and observed variables, may be specified to ensure sufficient power to conduct the analysis and to stay within the limits of sample size requirements. Model fit will be assessed using the $\chi^{2}$ goodness-of-fit test, Bollen's incremental fit index (0.95) and the standardised root mean residual $(0.08)$ values. Tests of mediation will be conducted using a resampling method. To test the significance of specific indirect effects of multiple mediators (total and specific for each mediator, using both normal theory and bootstrap procedures), a tool developed by Selig and Preacher ${ }^{92}$ will be used.

\section{Subgroup analysis}

Interactions between condition factors and baseline participant groupings (centre, gender, age, BMI at entry, $\% \mathrm{WL}$ at entry) will be prioritised as a priori subgroup analyses in the primary and secondary outcomes. Age, BMI and weight will be analysed as categorical variables to avoid assumptions of linearity (or the complexity of fitting polynomial terms) and for ease of presentation. These pre-planned analyses will be conducted by the inclusion of appropriate interaction terms in the regression models. Where interactions are significant, results for each subgroup will be reported. This will mitigate the problems of multiple testing due to many subgroup comparisons. We recognise that statistical power in subgroups will vary with sample size and provide a plot of detectable effect size versus sample size for reference (figure 3).

\section{Cost-effectiveness analyses}

Cost-effectiveness analysis will be conducted to model costs and intervention effects for a lifetime horizon. An existing decision-analytical model will be used to estimate BMI category transitions, incidence of secondary diseases (eg, type 2 diabetes), quality of life and healthcare costs. ${ }^{93}$ Quality-adjusted life-years and costs will allow calculation of incremental cost-effectiveness ratios and estimates of cost-utility. Extensive sensitivity analyses will test the modelling assumptions. Exploration of scenarios relating inter alia to the cost and effects (including sustainability) of the intervention. Secondary cost-effectiveness analysis will explore WLM outcomes (eg, cost per g maintained per year). The model will be adapted separately for each country.

Power calculations and sample size estimation

Power calculations were based on the primary outcome (weight change). To detect a difference between 


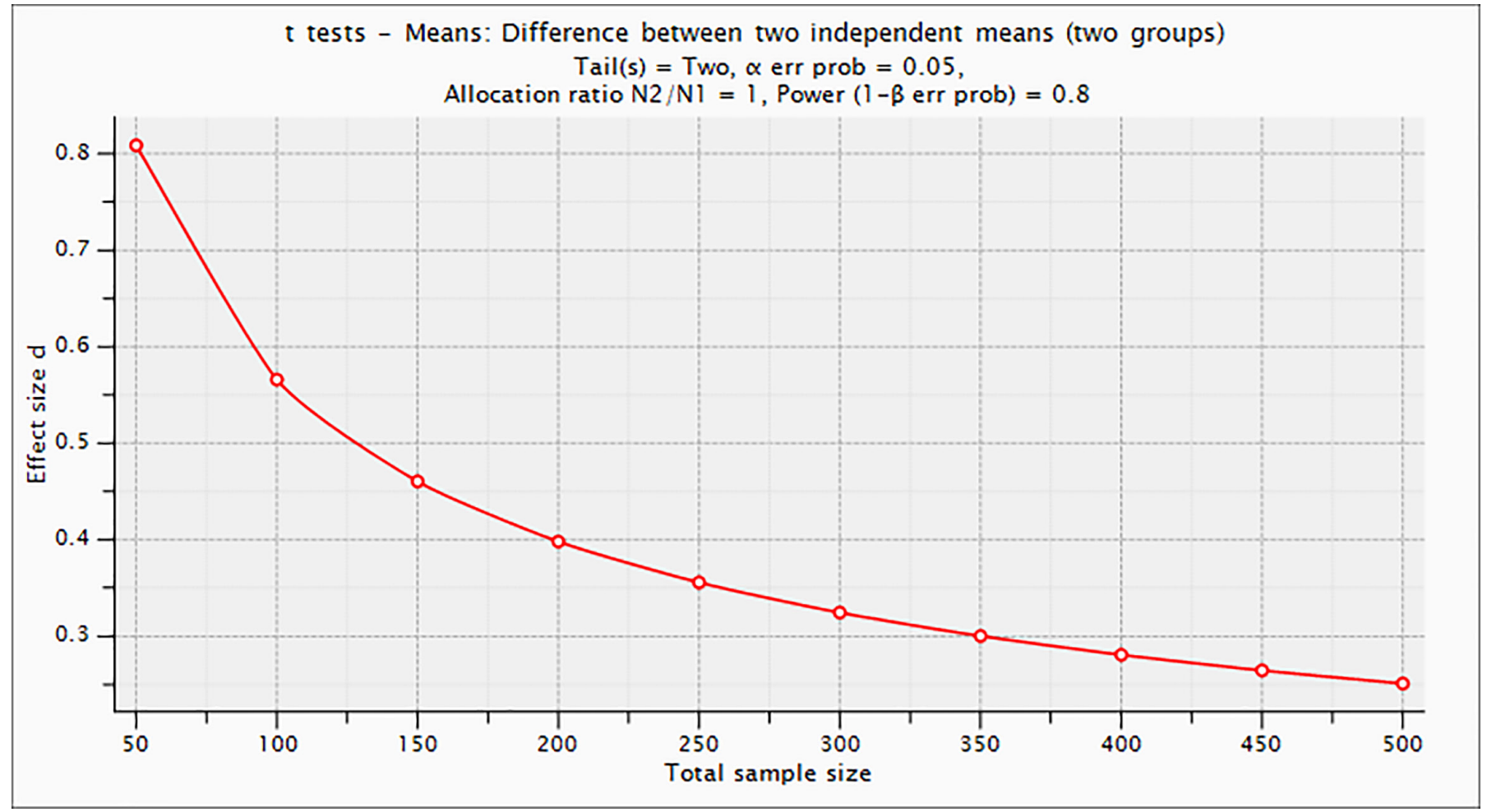

Figure 3 Plot of detectable effect size (Cohen's d).

treatment arms of $>1.5 \mathrm{~kg}$ body weight gives a (Cohen's) $\mathrm{d}$ value of $0.25 .^{22}$ For $80 \%$ power, comparing more than two groups requires a sample size of 250 per trial arm. To detect an effect size of 0.25 SD units for HbA1c, 245 participants in each trial arm gives $80 \%$ power, at $5 \%$ significance. Assuming $38 \%$ dropout, ${ }^{83}$ a sample size of 1600 (533 per centre) is needed to achieve a sample of 1002 (334 per centre, 250 per trial arm) participants at 12 months.

\section{Ethics and dissemination}

The trial was registered with the ISRCTN registry (ISRCTN88405328). Ethical approval was granted by each institutional ethics committee before study commencement at each centre. The protocol complies with relevant EC legislation, international conventions and declarations relating to ethical research practices. ${ }^{94}$ Interventions will be discontinued if they are reported detrimental. Protocol deviations, violations and serious adverse events or adverse events are recorded by trial staff and monitored by the principal investigator (RJS) and TSC. ${ }^{95}$ Any protocol amendments that impact the study conduct are approved by each institutional ethics committee and communicated to participants. Trial results will be published in peer-reviewed scientific journals. Baseline data will be analysed after the end of recruitment (March 2018) and outcome analyses after 1-year data are collected (March 2019). Authorship will be determined by the guidelines set out by the International Committee of Medical Journal Editors.

\section{POTENTIAL OUTCOMES AND IMPACT}

This is the first project to develop and formally evaluate (via randomised controlled trial) a new ICT toolkit combining continuous tracking of physical activity and body weight with theoretically informed, evidence-based digital interventions targeting self-regulation and motivation, and CB-emotion regulation in a $2 \times 2$ design, to target long-term weight management.

The NoHoW toolkit comprises evidence-based behaviour change techniques and CB-emotion regulation strategies, which are hypothesised mechanisms of action that may impact EB behaviours and longer term weight outcomes. Analyses of primary outcomes will enable us to test the hypotheses that self-regulation of EB behaviours improves longer term weight outcomes and that CB-emotion regulation strategies help prevent weight relapse. Secondary outcomes of the trial moderator effects will enable us to determine if different psychological and behavioural profiles of participants predict response to self-regulation or emotion regulation interventions. Mediation analyses will enable us to determine the mechanisms of action by which self-regulation and emotion regulation-based behaviour change approaches affect EB behaviours and longer term weight and health. Secondary analyses will inform more personalised digital interventions that better match intervention content and delivery to individual user needs, potentially helping to improve user engagement, health and well-being of participants. 


\section{Limitations}

Participants are blinded to study allocation, but they can access similar interventions from other real-world sources. The $2 \times 2$ design involved an active control. A $2 \times 2$ plus one design including a no-intervention control would have enabled evaluation of self-tracking using Fitbit devices versus no intervention.

The active control arm includes self-monitoring of physical activity and weight but the inclusion of the Fitbit devices across all arms potentially contaminates or diminishes the effect of self-regulatory components.

There is a potential conflict inherent in the design of trial that seeks to allow a certain degree of personalisation of intervention content use and maintain the integrity of trial arms. For this reason, the degree of personalisation was constrained to choice in the use of available self-regulatory or contextual emotion regulatory content within respective trial arms.

Social support was deliberately not part of this intervention but it is known that the degree of social support can be important for weight management.

\section{Author affiliations}

${ }^{1}$ School of Psychology, Faculty of Medicine and Health, University of Leeds, Leeds, UK

${ }^{2}$ Center for Research in Neuropsychology and Cognitive Behavioral Intervention,

University of Coimbra, Coimbra, Portugal

${ }^{3}$ Faculty of Human Kinetics, University of Lisbon, Lisbon, Portugal

${ }^{4}$ Institute of Health \& Society, Newcastle University, Newcastle upon Tyne, UK

${ }^{5}$ VTT, VTT Technical Research Centre of Finland, Ltd, Espoo, Finland

${ }^{6}$ Research Unit for Dietary Studies, Bispebjerg and Frederiksberg Hospital, The

Parker Institut, Frederiksberg, The Capital Region, Denmark

${ }^{7}$ Department of Public Health, University of Copenhagen, Copenhagen, Denmark

${ }^{8}$ BioSS, Biomathematics and Statistics Scotland, Aberdeen, UK

${ }^{9}$ Trinity Centre for Practice and Healthcare Innovation \& ADAPT Centre, University of Dublin Trinity College, Dublin, UK

${ }^{10}$ College of Life and Natural Sciences, University of Derby, Derby, UK

Contributors RJS, BLH, PJT, FFS, ALP and GH conceived the study. RJS is the principle investigator; $B L H$ is the grant coordinator; and SES and CD are the trial managers. SCL, ALP and SES are site coordinators. GH is the trial statistician and leads data management. MH and EM are responsible for the technological development and management of the NoHoW digital toolkit. ALP, MMM, MM and $\mathrm{CD}$ developed the content of the arms of the digital toolkit. JE, IS, MLM, CS, BP and LR conducted the trial activities at each respective trial sites. EHE and KS were involved in the development of some of the trial outcome measures. SES, CD and RJS drafted the manuscript and all authors revised and approved the final version. All authors made substantial contributions to the conceptualisation of the study design and conduct of the protocol.

Funding This project has received funding from the European Union's Horizon 2020 research and innovation programme under grant agreement №. 643309. The material presented and views expressed here are the responsibility of the author(s) only. The EU Commission takes no responsibility for any use made of the information set out. MMM is funded by the Marie-Sklodowska-Curie (EDGE) Fellowship programme (grant agreement No. 713567).

Competing interests RJS consults for Slimming World through the University of Leeds. MMM and GH also conducted consultancy work with Slimming World. Slimming World were a partner of the project to aid recruitment within the UK but will not be involved in the analysis and interpretation of the trial.

Patient consent for publication Not required.

Provenance and peer review Not commissioned; externally peer reviewed.

Data availability statement All data relevant to the study are included in the article or uploaded as supplementary information.
Open access This is an open access article distributed in accordance with the Creative Commons Attribution 4.0 Unported (CC BY 4.0) license, which permits others to copy, redistribute, remix, transform and build upon this work for any purpose, provided the original work is properly cited, a link to the licence is given, and indication of whether changes were made. See: https://creativecommons.org/ licenses/by/4.0/.

ORCID iD

Sarah Ellen Scott http://orcid.org/0000-0002-8168-7064

\section{REFERENCES}

1. Hunt A, Ferguson J. Health costs in the European Union: how much is related to EDCS. Brussels, the health and environmental alliance, 2014. Available: https://www.env-health.org/IMG/pdf/18062014 final_health_costs_in_the_european_union_how_much_is_realted_to_ edcs.pdf [Accessed 04 Jan 2019].

2. World Health Organization. The challenge of obesity in the who European region and the strategies for response. Copenhagen, Denmark Europe, 2007. Available: http://www.euro.who.int/_data/ assets/pdf_file/0008/98243/E89858.pdf [Accessed 04 Jan 2019].

3. Wang YC, McPherson K, Marsh T, et al. Health and economic burden of the projected obesity trends in the USA and the UK. The Lancet 2011;378:815-25.

4. Santos I, Sniehotta FF, Marques MM, et al. Prevalence of personal weight control attempts in adults: a systematic review and metaanalysis. Obesity Reviews 2017;18:32-50.

5. Franz MJ, VanWormer JJ, Crain AL, et al. Weight-Loss outcomes: a systematic review and meta-analysis of weight-loss clinical trials with a minimum 1-year follow-up. J Am Diet Assoc 2007;107:1755-67.

6. Brownell KD, Marlatt GA, Lichtenstein E, et al. Understanding and preventing relapse. American Psychologist 1986;41:765-82.

7. Stubbs RJ, Gail C, Whybrow S, et al. The evolutionary inevitability of obesity in modern society: implications for behavioral solutions to weight control in the general population. In: Martinez MP, Robinson $\mathrm{H}$, eds. Obesity and weight management: challenges, practices and health implications. New York: Novo Publishers, 2012.

8. Sainsbury K, Evans EH, Pedersen S, et al. Attribution of weight regain to emotional reasons amongst European adults with overweight and obesity who regained weight following a weight loss attempt. Eat Weight Disord 2018:1-11 (published Online First: 16 February 2018).

9. Stubbs RJ, Lavin JH. The challenges of implementing behaviour changes that lead to sustained weight management. Nutrition Bulletin 2013;38:5-22.

10. Dombrowski SU, Avenell A, Sniehott FF. Behavioural interventions for obese adults with additional risk factors for morbidity: systematic review of effects on behaviour, weight and disease risk factors. Obes Facts 2010;3:377-96.

11. Michie $S$, Abraham $C$, Whittington $C$, et al. Effective techniques in healthy eating and physical activity interventions: a meta-regression. Health Psychology 2009;28:690-701.

12. Teixeira PJ, Going SB, Sardinha LB, et al. A review of psychosocial pre-treatment predictors of weight control. Obesity Reviews 2005;6:43-65.

13. Silva MN, Markland D, Carraca EV, et al. Exercise autonomous motivation predicts 3-yr weight loss in women. Medicine \& Science in Sports \& Exercise 2011;43:728-37.

14. Kwasnicka D, Presseau J, White M, et al. Does planning how to cope with anticipated barriers facilitate health-related behaviour change? A systematic review. Health Psychol Rev 2013;7:129-45.

15. Dombrowski SU, Sniehotta FF, Avenell A, et al. Identifying active ingredients in complex behavioural interventions for obese adults with obesity-related co-morbidities or additional risk factors for comorbidities: a systematic review. Health Psychol Rev 2012;6:7-32.

16. Silva MN, Vieira PN, Coutinho SR, et al. Using self-determination theory to promote physical activity and weight control: a randomized controlled trial in women. J Behav Med 2010;33:110-22.

17. Teixeira PJ, Mata J, Williams GC, et al. Self-Regulation, motivation, and psychosocial factors in weight management. $J$ Obes 2012;2012:1-4.

18. Teixeira PJ, Carraça EV, Marques MM, et al. Successful behavior change in obesity interventions in adults: a systematic review of selfregulation mediators. BMC Med 2015;13:84.

19. Hagger MS, Chatzisarantis NLD. An integrated behavior change model for physical activity. Exerc Sport Sci Rev 2014;42:62-9.

20. Teixeira PJ, Silva MN, Mata J, et al. Motivation, self-determination, and long-term weight control. Int J Behav Nutr Phys Act 2012;9. 
21. Verstuyf $\mathrm{J}$, Patrick $\mathrm{H}$, Vansteenkiste $\mathrm{M}$, et al. Motivational dynamics of eating regulation: a self-determination theory perspective. Int $J$ Behav Nutr Phys Act 2012;9.

22. Dombrowski SU, Knittle K, Avenell A, et al. Long term maintenance of weight loss with non-surgical interventions in obese adults: systematic review and meta-analyses of randomised controlled trials. BMJ 2014;348:92646.

23. O'Reilly GA, Cook L, Spruijt-Metz D, et al. Mindfulness-based interventions for obesity-related eating behaviours: a literature review. Obes Rev 2014;15:453-61.

24. Dallman MF, Pecoraro N, Akana SF, et al. Chronic stress and obesity: A new view of "comfort food". Proc Natl Acad Sci U S A 2003;100:11696-701.

25. Block JP, $\mathrm{He} \mathrm{Y}$, Zaslavsky AM, et al. Psychosocial stress and change in weight among US adults. Am J Epidemiol 2009;170:181-92.

26. Gibson EL. The psychobiology of comfort eating: implications for neuropharmacological interventions. Behav Pharmacol 2012;23:442-60.

27. LeBel JL, Lu J, Dubé L. Weakened biological signals: Highlydeveloped eating schemas amongst women are associated with maladaptive patterns of comfort food consumption. Physiol Behav 2008;94:384-92.

28. Pedersen S, Sniehotta FF, Sainsbury K, et al. The complexity of selfregulating food intake in weight loss maintenance. A qualitative study among short- and long-term weight loss maintainers. Soc Sci Med 2018;208:18-24.

29. Hendershot CS, Witkiewitz K, George WH, et al. Relapse prevention for addictive behaviors. Subst Abuse Treat Prev Policy 2011;6:17.

30. Bowen S, Chawla N, Witkiewitz K. Mindfulness-based relapse prevention for addictive behaviors. In: Baer RA, ed. MindfulnessBased Treatment Approaches: A Clinician's Guide. 2nd ed. San Diego, CA: Elsevier Academic Press, 2014: 141-57.

31. Duarte C, Stubbs J, Pinto-Gouveia J, et al. The impact of selfcriticism and self-reassurance on weight-related affect and well-being in participants of a commercial weight management programme. Obes Facts 2017;10:65-75.

32. Lillis J, Hayes SC, Bunting K, et al. Teaching acceptance and mindfulness to improve the lives of the obese: a preliminary test of a theoretical model. ann. behav. med. 2009;37:58-69.

33. Forman EM, Butryn ML, Hoffman KL, et al. An open trial of an acceptance-based behavioral intervention for weight loss. Cogn Behav Pract 2009;16:223-35.

34. Forman EM, Butryn ML, Juarascio AS, et al. The mind your health project: a randomized controlled trial of an innovative behavioral treatment for obesity. Obesity 2013;21:1119-26.

35. Niemeier HM, Leahey T, Palm Reed K, et al. An acceptance-based behavioral intervention for weight loss: a pilot study. Behav Ther 2012;43:427-35.

36. Tapper K, Shaw C, Ilsley J, et al. Exploratory randomised controlled trial of a mindfulness-based weight loss intervention for women. Appetite 2009:52:396-404.

37. Carrière K, Khoury B, Günak MM, et al. Mindfulness-based interventions for weight loss: a systematic review and meta-analysis. Obes Rev 2018;19:164-77.

38. Yardley L, Choudhury T, Patrick K, et al. Current issues and future directions for research into digital behavior change interventions. $A m$ J Prev Med 2016;51:814-5.10.1016/j.amepre.2016.07.019

39. MacLean PS, Wing RR, Davidson T, et al. Nih Working Group report: innovative research to improve maintenance of weight loss. Obesity 2015;23:7-15.

40. Evans EH, Araújo-Soares V, Adamson A, et al. The NULevel trial of a scalable, technology-assisted weight loss maintenance intervention for obese adults after clinically significant weight loss: study protocol for a randomised controlled trial. Trials 2015;16:421.

41. Lentferink AJ, Oldenhuis HKE, de Groot M, et al. Key components in eHealth interventions combining Self-Tracking and persuasive eCoaching to promote a healthier lifestyle: a scoping review. J Med Internet Res 2017:19:e277.

42. European Commission. eHealth action plan 2012-2020, 2012. Available: http://ec.europa.eu/digital-agenda/en/news/ehealthaction-plan-2012-2020-innovative-healthcare-21st-century [Accessed 4 Jan 2019].

43. Pagoto S, Schneider K, Jojic M, et al. Evidence-Based strategies in weight-loss mobile Apps. Am J Prev Med 2013;45:576-82.

44. Breton ER, Fuemmeler BF, Abroms LC. Weight loss - there is an APP for that! but does it adhere to evidence-informed practices? Trans/ Behav Med 2011;1:523-9.10.1007/s13142-011-0076-5

45. Hutchesson MJ, Rollo ME, Krukowski R, et al. eHealth interventions for the prevention and treatment of overweight and obesity in adults: a systematic review with meta-analysis. Obes Rev 2015;16:376-92.
46. Chan A-W, Tetzlaff JM, Altman DG, et al. Spirit 2013 statement: defining standard protocol items for clinical trials. Ann Intern Med 2013;158:200-7.

47. Klem ML, Wing RR, McGuire MT, et al. A descriptive study of individuals successful at long-term maintenance of substantial weight loss. Am J Clin Nutr 1997;66:239-46.

48. Thomas S, Reading J, Shephard RJ. Revision of the physical activity readiness questionnaire (PAR-Q). Can J Sport Sci 1992;17:338-45.

49. Altman DG, Bland JM. Treatment allocation by minimisation. BMJ 2005;330.

50. Craig P, Dieppe P, Macintyre S, et al. Developing and evaluating complex interventions: the new medical Research Council guidance. Int J Nurs Stud 2013;50:587-92.

51. De Lorenzo A, Andreoli A, Matthie J, et al. Predicting body cell mass with bioimpedance by using theoretical methods: a technological review. J Appl Physiol 1997;82:1542-58.

52. World Health Organisation. Guide to physical measurements (step 2), 2008. Available: https://www.who.int/ncds/surveillance/steps/ Section\%204\%20Step\%202\%20Physical\%20Measurements.pdf [Accessed 4 Jan 2019].

53. Jain A, Rao N, Sharifi M, et al. Evaluation of the point of care Afinion AS100 analyser in a community setting. Ann Clin Biochem 2017;54:331-41

54. Van Uum SHM, Sauvé B, Fraser LA, et al. Elevated content of cortisol in hair of patients with severe chronic pain: a novel biomarker for stress. Stress 2008;11:483-8.

55. Craig CL, Marshall AL, Sjorstrom M, et al. International physical activity questionnaire: 12 -country reliability and validity. Medicine \& Science in Sports \& Exercise 2003;35:1381-95.

56. Mullen SP, Silva MN, Sardinha LB, et al. Initial validation of the activity choice index among overweight women. Res Q Exerc Sport 2016;87:174-81.

57. Simpson E, Bradley J, Poliakov I, et al. Iterative development of an online dietary recall tool: INTAKE24. Nutrients 2017;9:118.

58. Stunkard AJ, Messick S. The three-factor eating questionnaire to measure dietary restraint, disinhibition and hunger. J Psychosom Res 1985;29:71-83

59. Arnold TA, Johnston CS, Lee CD, et al. Eating in the absence of hunger in college students. Appetite 2015;92:51-6.

60. Tylka TL, Kroon Van Diest AM. The intuitive eating Scale-2: item refinement and psychometric evaluation with College women and men. J Couns Psychol 2013;60:137-53.

61. Gormally J, Black S, Daston S, et al. The assessment of binge eating severity among obese persons. Addict Behav 1982;7:47-55.

62. Herdman M, Gudex C, Lloyd A, et al. Development and preliminary testing of the new five-level version of EQ-5D (EQ-5D-5L). Qual Life Res 2011;20:1727-36.

63. Tennant R, Hiller L, Fishwick R, et al. The Warwick-Edinburgh mental well-being scale (WEMWBS): development and UK validation. Health Qual Life Outcomes 2007;5:63.

64. Sniehotta FF, Schwarzer R, Scholz U, et al. Action planning and coping planning for long-term lifestyle change: theory and assessment. Eur J Soc Psychol 2005;35:565-76.

65. Sniehotta FF, Scholz U, Schwarzer R. Bridging the intentionbehaviour gap: planning, self-efficacy, and action control in the adoption and maintenance of physical exercise. Psychol Health $2005 ; 20: 143-60$

66. JY N, Ntoumanis N, Thøgersen-Ntoumani C, et al. Predicting psychological needs and well-being of individuals engaging in weight management: the role of important others. Appl Psychol Health Well Being 2013:5:291-310.

67. Sebire SJ, Standage M, Vansteenkiste M. Development and validation of the goal content for exercise questionnaire. $J$ Sport Exer Psychol 2008;30:353-77.

68. Markland D, Ingledew DK. The measurement of exercise motives: factorial validity and invariance across gender of a revised exercise motivations inventory. Br J Health Psychol 1997;2:361-76.

69. Markland D, Tobin V. A modification to the behavioural regulation in exercise questionnaire to include an assessment of Amotivation. $J$ Sport Exer Psychol 2004;26:191-6.

70. Pelletier LG, Dion SC, Slovinec-D'Angelo M, et al. Why do you regulate what you eat? relationships between forms of regulation, eating behaviors, sustained dietary behavior change, and psychological adjustment. Motiv Emot 2004;28:245-77.

71. Palmeira AL, Teixeira PJ, Branco TL, et al. Predicting short-term weight loss using four leading health behavior change theories. Int $J$ Behav Nutr Phys Act 2007;4.

72. Gilbert $\mathrm{P}, \mathrm{Clarke} \mathrm{M}, \mathrm{Hempel} \mathrm{S}$, et al. Criticizing and reassuring oneself: an exploration of forms, styles and reasons in female students. Br J ClinPsychol 2004;43:31-50. 
73. Goss K, Gilbert P, Allan S. An exploration of shame measures-I: the other as Shamer scale. Pers Individ Dif 1994;17:713-7.

74. Allan S, Gilbert P, Goss K. An exploration of shame measures-II: psychopathology. Pers Individ Dif 1994;17:719-22.10.1016/01918869(94)90150-3

75. Gilbert P, Catarino F, Duarte C, et al. The development of compassionate engagement and action scales for self and others. $J$ of Compassionate Health Care 2017;4

76. Sandoz EK, Wilson KG, Merwin RM, et al. Assessment of body image flexibility: the body image-acceptance and action questionnaire. Journal of Contextual Behavioral Science 2013;2:39-48.

77. Trompetter HR, ten Klooster PM, Schreurs KMG, et al. Measuring values and committed action with the engaged living scale (ELS): psychometric evaluation in a nonclinical sample and a chronic pain sample. Psychol Assess 2013;25:1235-46.

78. Carlson LE, Brown KW. Validation of the mindful attention awareness scale in a cancer population. J Psychosom Res 2005;58:29-33.

79. Gratz KL, Roemer L. Multidimensional assessment of emotion regulation and dysregulation: development, factor structure, and initial validation of the difficulties in emotion regulation scale. $J$ Psychopathol Behav Assess 2004;26:41-54.

80. Fresco DM, Moore MT, van Dulmen MHM, et al. Initial psychometric properties of the experiences questionnaire: validation of a selfreport measure of decentering. Behav Ther 2007;38:234-46.

81. Cohen S, Kamarck T, Mermelstein R. A global measure of perceived stress. J Health Soc Behav 1983;24:385-96.

82. Antony MM, Bieling PJ, Cox BJ, et al. Psychometric properties of the 42-item and 21-item versions of the depression anxiety stress scales in clinical groups and a community sample. Psychol Assess 1998;10:176-81.

83. Larsen TM, Dalskov S, van Baak M, et al. The diet, obesity and genes (Diogenes) dietary study in eight European countries â€" a comprehensive design for long-term intervention. Obes Rev 2010;11:76-91.

84. Cappelleri JC, Bushmakin AG, Gerber RA, et al. Evaluating the power of food scale in obese subjects and a general sample of individuals: development and measurement properties. Int J Obes 2009;33:913-22.

85. Weinstein N, Przybylski AK, Ryan RM. The index of autonomous functioning: development of a scale of human autonomy. J Res Pers 2012;46:397-413.

86. Grouzet FME, Kasser T, Ahuvia A, et al. The structure of goal contents across 15 cultures. J Pers Soc Psychol 2005;89:800-16.

87. Tylka TL. Development and psychometric evaluation of a measure of intuitive eating. J Couns Psychol 2006;53:226-40.

88. Fuglestad PT, Rothman AJ, Jeffery RW, et al. Regulatory focus, proximity to goal weight, and weight loss maintenance. Am J Health Behav 2015;39:709-20.

89. Kaasinen E, Mattila E, Lammi H, et al. Technology acceptance model for mobile services as a design framework. In: Lumsden J, ed. Human-Computer interaction and innovation in handheld, mobile and wearable technologies. Hershey: IGI Global, 2011: 80-107.

90. Nguyen J, Moorhouse M, Curbow B, et al. Construct validity of the eHealth literacy scale (eHEALS) among two adult populations: a Rasch analysis. JMIR Public Health Surveill 2016;2.

91. Ritchie J, Spencer L, O'Connor W. Carrying out qualitative analysis. In: Richie J, Lewis J, eds. Qualitative research practice. London: Sage Publications, 2004: 219-62.

92. Preacher KJ, Selig JP. Monte Carlo method for assessing multilevel Mediation: An interactive tool for creating confidence intervals for indirect effects in 1-1-1 multilevel models [Computer software]. Available: http://quantpsy.org/

93. Meads DM, Hulme CT, Hall P, et al. The cost-effectiveness of primary care referral to a UK commercial weight loss programme. Clin Obes 2014:4:324-32.

94. General Assembly of the World Medical Association. World Medical association Declaration of Helsinki: ethical principles for medical research involving human subjects. J Am Coll Dent 2014;81.

95. Medical Research Council. MRC guidelines for management of global health trials - Involving Clinical or Public Health Interventions. MRC, 2017. Available: https://mrc.ukri.org/documents/pdf/ guidelines-for-management-of-global-health-trials/ [Accessed 4 Jan 2019]. 